\title{
O Ensino da Programação e o Desenvolvimento do Pensamento Lógico: uma Revisão Sistemática de Literatura
}

\section{The Teaching of Programming and the Development of Logical Thinking: a Systematic Literature Review}

\author{
Rodrigo Shimasakia; Maria Elisabette Brisola Brito Prado ${ }^{\mathrm{ab}}$
}

\begin{abstract}
${ }^{a}$ Unopar, Programa de Pós-Graduação Stricto Sensu em Metodologias para Ensino de Linguagens e suas Tecnologias. PR, Brasil.
bUniversidade Anhanguera de São Paulo, Programas de Pós-Graduação Stricto Sensu em Educação Matemática. SP, Brasil

*E-mail: rshimasaki@gmail.com
\end{abstract}

\begin{abstract}
Resumo
Este artigo apresenta um estudo sobre a Lógica de Programação voltada para o contexto de ensino e aprendizagem por meio de uma revisão sistemática de literatura. Considerando que a tecnologia se faz presente em todas as atividades do cotidiano, torna-se cada vez mais evidenciada a necessidade de propiciar o desenvolvimento do pensamento crítico, lógico e científico dos estudantes. Assim, busca-se identificar e analisar artigos científicos publicados nos últimos seis anos no repositório do Simpósio Brasileiro de Informática na Educação (SBIE). O método utilizado para coleta de dados, com definição de critério de inclusão e exclusão, permitiu a identificação de dezoito artigos que tratam de pesquisas sobre a Lógica da Programação nos três níveis de Educação do sistema brasileiro, ou seja, do Ensino Fundamental, Ensino Médio e Ensino Superior. O resultado da análise dos artigos mostra que tanto no Ensino Fundamental como no Ensino Médio há uma tendência em propiciar ao aluno vivenciar uma nova forma de aprender com o uso das tecnologias computacionais que permite explorar o pensamento lógico por meio de jogos e da atividade de programação, como o Scratch. No Ensino Superior existe a preocupação em utilizar as tecnologias para inovar as Metodologias de Ensino visando o engajamento do estudante. Nesse sentido, o destaque é dado nos ambientes virtuais de aprendizagem, os sistemas de tutores inteligentes e gamificação.
\end{abstract}

Palavras-chave: Lógica de Programação. Scratch. Gamificação.

\begin{abstract}
This article presents a study on Logic Programming in the teaching and learning context through a systematic literature review. Considering that technology is present in all the daily life activities, it becomes increasingly evident the need to foster the development of students' critical, logical, and scientific thinking. Thus, it was sought to identify and analyze scientific articles published in the last six years in the repository of the Brazilian Symposium of Informatics in Education (Simpósio Brasileiro de Informática na Educação - SBIE). The method used for data collection, with the definition of inclusion and exclusion criteria, allowed the identification of 18 articles dealing with research on Programming Logic in the three levels of education of the Brazilian system, that is, elementary school, high school, and higher education. The result of the articles analysis shows that in both elementary and high school there is a tendency to provide students with a new way of learning through the use of computer technologies that allow them to explore logical thinking through games and through the Scratch programming activity. In higher education there is a concern in using technologies to innovate teaching methodologies, aiming at student engagement. Thus, the emphasis is on virtual learning environments, intelligent tutoring systems, and gamification.
\end{abstract}

Keywords: Programming Logic. Scratch. Gamification.

\section{Introdução}

A atividade de programação no âmbito da Educação teve início no Brasil nos anos 1980, com advento dos computadores pessoais (Personal Computer - PC). Um dos grandes precursores da utilização da programação na Educação Básica foi o educador e matemático Seymour Papert, criador da linguagem de programação Logo. Para Papert (1985), é a criança quem deve programar o computador e, dessa forma, dominar suas ações em relação ao computador, ou seja, ela quem decide o que a máquina irá fazer. Este sentimento de domínio é que vai estabelecer um contato maior com algumas ideias mais profundas da Ciência e da Arte de construir modelos intelectuais.

$\mathrm{O}$ autor enfatiza que os computadores deveriam ser utilizados para que os estudantes pudessem "pensar com" as máquinas e "pensar sobre" o próprio pensar. Sob este enfoque, a criação da linguagem de Logo teve como base os princípios educacionais presentes na teoria de Papert (1994), denominada Construcionismo. Essa teoria denominada de Construcionismo considera a importância de o estudante aprender de forma ativa e reflexiva, construindo algo (no caso, um programa) que lhe seja significativo, de modo que possa se envolver, afetiva e cognitivamente, com aquilo que está sendo produzido.

Esse potencial da atividade de programação, na época, norteou a implementação da linguagem Logo em algumas escolas públicas e privadas do Ensino Básico no país. Entretanto, somente nos últimos anos que a programação 
vem ganhando novo impulso para ser utilizada nos vários níveis de escolaridade. A par deste movimento se observa que a evolução da tecnologia tem favorecido esse processo, tornando a atividade de programação mais acessível para este público de estudantes, por meio da linguagem Scratch, do Arduino e da Robótica, por exemplo. Vale ressaltar que o uso destes recursos permite ao aluno vivenciar uma nova forma de aprender, aplicando conceitos e estratégias de resolução de problemas, refletindo e depurando suas ideias, assim como desenvolvendo o raciocínio lógico.

Outro fato importante, que tem contribuído nesse processo crescente da programação, nas práticas escolares, são as diretrizes expressas nos documentos oficiais brasileiros, como por exemplo, a Base Nacional Comum Curricular - BNCC (Brasil, 2018), que destaca a importância de o estudante vivenciar a atividade de programação como meio favorável para desenvolver o pensamento computacional.

Autores, nacionais e internacionais, como Wing (2006), Ramos e Espadeiro, 2014, Valente (2016), Prado, Silva, Pietropaolo e Kfouri (2020) destacam que o pensamento computacional envolve abstração, lógica e representação de dados, assim como a construção da linguagem algorítmica relacionada com a resolução de problemas.

Para Puga e Rissetti (2009), um programa é um conjunto de instruções escrito em uma linguagem que diz ao computador o que deve ser feito. E esta linguagem é formada por palavras que, por sua vez, são agrupadas em frases para produzir um determinado significado.

Programar significa, pela ótica de Papert (1985, p. 18): "comunicar-se com o computador em uma linguagem que tanto ele quanto o homem podem "entender", pois o que está subjacente neste processo é a lógica. De fato, a lógica é fundamental nas atividades de programação e, segundo o dicionário Houaiss, refere-se a um "modo de raciocinar coerente que expressa uma relação de causa e consequência". Para os autores Forbellone e Eberspacher (2005), a lógica de programação significa que o uso correto das leis do pensamento e de processos de raciocínio e simbolização na programação de computadores cooperem para a produção de soluções logicamente válidas e coerentes, que resolvam com qualidade os desejos que se deseja programar.

Considerando a pertinência dessa temática, Lógica de Programação, atualmente, se busca neste artigo realizar um estudo para identificar e analisar pesquisas realizadas envolvendo o ensino da lógica de programação, a partir das etapas da Revisão Sistemática de Literatura.

\section{Desenvolvimento}

\subsection{Metodologia}

A Revisão Sistemática de Literatura (RSL), segundo os autores Sampieri, Collado e Lucio (2013), trata de "uma estratégia de intervenção específica, mediante a aplicação de métodos explícitos e sistematizados de busca, apreciação crítica e síntese da informação selecionada" (p. 84). A RSL visa ampliar o campo de estudo para que o pesquisador consiga explorar o assunto até que se alcance um referencial cientificamente seguro e confiável, que leve à validação da pesquisa.

No processo de revisão sistemática de literatura, é imprescindível que sejam registadas todas as etapas de pesquisa, não só para que esta possa ser replicável por outro investigador, como também para se aferir que o processo em curso segue uma série de etapas previamente definidas e absolutamente respeitadas nas várias etapas (RAMOS; FARIA; FARIA, 2014, p.7).

Dessa forma se realiza uma análise preliminar de acordo com os critérios de escolha dos artigos seguindo as seguintes etapas:

Etapa 1- Identificar a problemática: vivencia-se um momento em que a tecnologia se faz presente em todas as atividades do cotidiano e que, além das competências que se adquire na escola como, por exemplo, argumentação, conhecimento, pensamento crítico e científico, também se verifica a necessidade do Pensamento Computacional que por meio de conhecimentos em computação e lógica possibilita aos alunos a solução de problemas de relativos ao seu cotidiano.

Etapa 2- Escolha do repositório de artigos: a realização da RSL se centrou no repositório do Simpósio Brasileiro de Informática na Educação (SBIE) integrante do Congresso Brasileiro de Informática na Educação (CBIE) organizado pela Sociedade Brasileira de Computação (SBC). O CBIE busca promover e incentivar as trocas de experiências entre as comunidades científica, profissional, governamental e empresarial na área de Informática na Educação, e é voltado essencialmente a discutir a pesquisa e os desafios sobre a Educação, tendo como premissa a reflexão do uso das tecnologias digitais da informação e comunicação no ensino com vistas a melhoria da qualidade educacional.

Etapa 3- Estabelecer critérios para inclusão/exclusão dos artigos na pesquisa: definem-se os seguintes critérios para a escolha dos artigos a serem analisados:

1. Artigos publicados no período de 2014 a 2019 - últimos seis anos;

2. Somente artigos disponíveis no repositório do SBIE;

3. Publicações em português;

4. Palavras de busca: "Lógica" and "Programação"; e

5. Excluídas pesquisas que não abordam a temática pesquisada.

Etapa 4- Caracterizar os artigos: inicialmente, foram encontrados vinte e dois artigos no período de 2002 a 2019 e, seguindo o critério foram selecionados apenas aqueles que foram publicados nos últimos seis anos. Dessa forma, foram excluídos três artigos dos anos de 2002, 2007 e 2012 e um artigo intitulado "Robótica experimental com uma arquitetura pedagógica para montagem de um sistema de irrigação inteligente", que se distanciava do escopo do tema deste estudo. Esta busca de literatura resultou em dezoito artigos que compõe o escopo de análise do presente estudo. 
Etapa 5- Analisar e discutir os dados: nesta etapa da pesquisa se procura ler todos os artigos e separar as principais ideias sobre cada uma das temáticas envolvidas. Dessa forma, foram organizados os resultados em eixos temáticos, conforme será apresentado no tópico dos resultados e discussões.

Etapa 6- Demonstrar os resultados: ao realizar a discussão dos dados foi possível levantar informações que vieram corroborar com as teorias apresentadas na introdução e mostrar as novas perspectivas relacionadas ao Pensamento Computacional nos dias de hoje.

Etapa 7- Apresentar conclusões: por fim, apresentar as principais conclusões sobre os artigos pesquisados buscando deixar claro que os objetivos da pesquisa foram atendidos e que o estudo poderá possibilitar novas ideias e novos estudos relacionados ao Ensino da Programação e o Pensamento Computacional, uma vez que é um assunto que está em constante desenvolvimento e possui outras interfaces que poderão ser exploradas em pesquisas futuras.

\subsection{Resultados e Discussão}

Este tópico engloba as etapas seis e sete da RSL e são apresentados os resultados da pesquisa mostrando os dezoito artigos constituintes deste estudo, e que seguem descritas no quadro 1 com a devida identificação dos autores, ano de publicação e o tema.

Quadro 1 - Compilação de autores e temas sobre Lógica de Programação

\begin{tabular}{|c|c|c|}
\hline Autor & Ano & Tema \\
\hline $\begin{array}{l}\text { Erico Amaral, Alex Camargo, Marina Gomes, Cesar } \\
\text { Huegel Richa, Liliane Becker }\end{array}$ & 2014 & $\begin{array}{l}\text { Desenvolvendo o Raciocínio Lógico no Ensino Médio: uma } \\
\text { proposta utilizando a ferramenta Scratch }\end{array}$ \\
\hline Tainá Medeiros, Pedrina Brasil, Eduardo Aranha & 2014 & $\begin{array}{l}\text { Um framework para criação de jogos voltados para o ensino } \\
\text { de lógica de programação }\end{array}$ \\
\hline Narúsci Bastos, Diana Adamatti, Fernanda Antoniolo & 2015 & $\begin{array}{l}\text { Ensino de Lógica de Programação no Ensino Médio e suas } \\
\text { implicações na Neurociências }\end{array}$ \\
\hline Cárlisson Galdino, Sebastião Neto, Evandro Costa & 2015 & $\begin{array}{l}\text { KidCoder: uma Proposta de Ensino de Programação de } \\
\text { forma Lúdica }\end{array}$ \\
\hline Taciana Pontual Falcão, Rafael Barbosa & 2015 & $\begin{array}{l}\text { “Aperta o Play!” Análise da Interação Exploratória em um } \\
\text { Jogo Baseado em Pensamento Computacional }\end{array}$ \\
\hline Marcella Café, Felipe Costa, Victor Silva, Daniela Terra & 2016 & $\begin{array}{l}\text { Lord of Code: Uma Ferramenta de Apoio ao Ensino da } \\
\text { Programação }\end{array}$ \\
\hline Leandro Nagano e Alexandre Direne. & 2016 & $\begin{array}{l}\text { Ensino de Lógica de Programação baseado na indução- } \\
\text { dedução através de exemplos }\end{array}$ \\
\hline $\begin{array}{l}\text { Lia Alessandra da S. Martins, Amanda da S. Brelaz, } \\
\text { Gabriel R. Nascimento, Raíssa M. Alfaia e Thaís dos S. } \\
\text { Martins }\end{array}$ & 2016 & $\begin{array}{l}\text { Ensinando Lógica de Programação aplicada a Robótica para } \\
\text { alunos do Ensino Fundamental }\end{array}$ \\
\hline $\begin{array}{l}\text { Erico Amaral, Alex Camargo, Marina Gomes, Cesar } \\
\text { Huegel Richa, Liliane Becker }\end{array}$ & 2017 & $\begin{array}{l}\text { ALGO+ Uma ferramenta para o apoio ao ensino de } \\
\text { Algoritmos e Programação para alunos iniciantes }\end{array}$ \\
\hline Brenda Barbosa, Sandro Silva, Bruno Sousa & 2017 & $\begin{array}{l}\text { Tri-Logic Proposta Lúdica Gamificada para o Ensino e } \\
\text { Aprendizagem da Lógica de Programação com o Uso } \\
\text { da Mineração de Dados como Ferramenta de Auxílio ao } \\
\text { Professor }\end{array}$ \\
\hline Stephanie Ribeiro, Amanda Melo & 2017 & $\begin{array}{l}\text { Um Método para o Desenvolvimento de Software com } \\
\text { Crianças Utilizando o Ambiente Scratch }\end{array}$ \\
\hline $\begin{array}{l}\text { Carla Rodriguez, Denise Goya, Mirtha Lina Fernandéz } \\
\text { Venero, Cláudia Abrão }\end{array}$ & 2017 & $\begin{array}{l}\text { Met@ Aprendiz@gem: estratégias metacognitivas } \\
\text { e recursos pedagógicos para apoiar a construção do } \\
\text { pensamento lógico }\end{array}$ \\
\hline $\begin{array}{l}\text { Glívia Barbosa, Ismael Santana, Flávio Coutinho, Pedro } \\
\text { Froes, Rafael Barbosa, Felipe Freitas, Vitória M. P. do } \\
\text { Nascimento, Mariana Bulgarelli, Gustavo Marques, João } \\
\text { Renan, Paula Ribeiro, Joice Sena, Daniel Franco }\end{array}$ & 2018 & $\begin{array}{l}\text { Play(code): uma proposta para estimular o aprendizado de } \\
\text { Lógica de Programação }\end{array}$ \\
\hline $\begin{array}{l}\text { Carla Rodriguez, Rafaela Vilela da Rocha, Denise Goya, } \\
\text { Mirtha Fernández Venero, Francisco Zampirolli }\end{array}$ & 2018 & $\begin{array}{l}\text { Critérios para inserção de estratégias cognitivas e } \\
\text { metacognitivas no desenvolvimento de lógica de } \\
\text { programação em ambientes virtuais de aprendizagem }\end{array}$ \\
\hline Graziela Guarda, Ione Goulart & 2018 & $\begin{array}{l}\text { Jogos Lúdicos sob a ótica do Pensamento Computacional: } \\
\text { Experiências do Projeto Logicamente }\end{array}$ \\
\hline Luiza Engler Stadelhofer, Isabela Gasparini & 2018 & $\begin{array}{l}\text { Ensino de Algoritmos e Lógica de Programação para } \\
\text { os Diferentes Cursos: Um Mapeamento Sistemático da } \\
\text { Literatura }\end{array}$ \\
\hline Êrica Peters do Carmo, Isabela Gasparini, Elaine Oliveira & 2019 & $\begin{array}{l}\text { Captura e Visualizacão das Trajetórias de Aprendizagem } \\
\text { como Ferramentas para a Análise do Comportamento dos } \\
\text { Estudantes em um Ambiente Adaptativo Educacional }\end{array}$ \\
\hline $\begin{array}{l}\text { Renata Barros, Orivaldo Santana Jr, Igor Silva, Luana dos } \\
\text { Santos, Vilson Rodrigues Câmara Neto }\end{array}$ & 2019 & $\begin{array}{l}\text { Predição do rendimento dos alunos em lógica de } \\
\text { programação com base no desempenho das disciplinas } \\
\text { do primeiro período do curso de Ciências e Tecnologia } \\
\text { utilizando técnicas de mineração de dados }\end{array}$ \\
\hline
\end{tabular}

Fonte: dados da pesquisa. 
O Quadro 2 mostra a quantidade dos artigos selecionados e o ano de publicação. Pode-se perceber que há um equilíbrio entre a quantidade de publicações ano a ano, ou seja, não há uma variabilidade grande em relação ao número de publicações.

Quadro 2 - Artigos por ano de publicação

\begin{tabular}{|c|c|}
\hline Ano de Publicação & Quantidade Publicada \\
\hline 2014 & 2 \\
\hline 2015 & 3 \\
\hline 2016 & 3 \\
\hline 2017 & 4 \\
\hline 2018 & 4 \\
\hline 2019 & 2 \\
\hline Total & $\mathbf{1 8}$ \\
\hline
\end{tabular}

Fonte: dados da pesquisa.

Entre os dezoito artigos selecionados se observa uma representatividade em várias regiões do país, sendo oito da Região Sul, quatro nas Regiões Sudeste e Nordeste, um nas Regiões Norte e Centro-oeste, como mostra o Quadro 3.

Quadro 3 - Artigos por Estado da federação

\begin{tabular}{|c|c|}
\hline Estado Brasileiro & Quantidade Publicação \\
\hline AL & 1 \\
\hline AM & 1 \\
\hline DF & 1 \\
\hline MG & 2 \\
\hline PE & 1 \\
\hline PR & 1 \\
\hline RN & 2 \\
\hline RS & 4 \\
\hline SC & 3 \\
\hline SP & 2 \\
\hline Total & $\mathbf{1 8}$ \\
\hline
\end{tabular}

Fonte: dados da pesquisa.

Ao realizar a leitura dos artigos se identifica a possibilidade de organizar análise sobre a Lógica de Programação em três níveis de escolaridade: Ensino Fundamental, Médio e Superior.

\section{Nível 1 - Lógica de programação no Ensino Fundamental}

Constata-se que entre os artigos selecionados, os autores de cinco desses (Quadro 4).

Quadro 4 - Artigos relacionados ao Ensino Fundamental

\begin{tabular}{|l|l|}
\hline \multicolumn{1}{|c|}{ Autores } & \multicolumn{1}{|c|}{ Tema } \\
\hline $\begin{array}{l}\text { Tainá Medeiros, Pedrina Brasil, } \\
\text { Eduardo Aranha }\end{array}$ & $\begin{array}{l}\text { Um framework para criação de } \\
\text { jogos voltados para o ensino de } \\
\text { lógica de programação }\end{array}$ \\
\hline $\begin{array}{l}\text { Taciana Pontual Falcão, Rafael } \\
\text { Barbosa }\end{array}$ & $\begin{array}{l}\text { “Aperta o Play!” Análise da } \\
\text { Interação Exploratória em um } \\
\text { Jogo Baseado em Pensamento } \\
\text { Computacional }\end{array}$ \\
\hline $\begin{array}{l}\text { Lia Alessandra da S. Martins, } \\
\text { Amanda da S. Brelaz, Gabriel R. } \\
\text { Nascimento, Raíssa M. Alfaia e } \\
\text { Thaís dos S. Martins }\end{array}$ & $\begin{array}{l}\text { Ensinando Lógica de } \\
\text { Programação aplicada a } \\
\text { Robótica para alunos do Ensino } \\
\text { Fundamental }\end{array}$ \\
\hline $\begin{array}{l}\text { Stephanie Ribeiro, Amanda } \\
\text { Melo }\end{array}$ & $\begin{array}{l}\text { Um Método para o } \\
\text { Desenvolvimento de Software } \\
\text { com Crianças Utilizando o } \\
\text { Ambiente Scratch }\end{array}$ \\
\hline Graziela Guarda, Ione Goulart & $\begin{array}{l}\text { Jogos Lúdicos sob a ótica do } \\
\text { Pensamento Computacional: } \\
\text { Experiências do Projeto } \\
\text { Logicamente }\end{array}$ \\
\hline Fonte: dados da pesquisa. &
\end{tabular}

Estudos para a utilização de jogos para o ensino de programação para crianças e, dessa forma, incentivar o pensamento lógico, a criatividade e a capacidade de resolução de problemas. Este foco voltado para o uso das tecnologias, na fase inicial da escolarização, foi defendido por Papert (1985, p.37), que criou a linguagem de programação Logo considerando que:

quando a criança aprende a programar, o processo de aprendizagem é transformado. O novo conhecimento é uma fonte de poder e é experimentado como tal a partir do momento que começa a formar na mente da criança.

Medeiros et al. (2014) apresentam, em seu artigo, como os jogos digitais estão sendo utilizados para abordar o conhecimento, possibilitando às crianças do nível básico ser a engrenagem motivadora que ajuda no processo de aprendizagem de diversas áreas do conhecimento. Com a popularização dos aparatos tecnológicos, principalmente, dos smartphones, as crianças estão tendo contato cada vez mais cedo com estes aparelhos e aprendem rápido como usá-los, mas dificilmente aprendem como eles funcionam e como podem explorar para desenvolver novas habilidades cognitivas.

Aprender a codificar não é uma tarefa fácil, especialmente, para as crianças e montar uma infraestrutura na escola para ensinar as crianças a programarem é mais complexa. Os autores propuseram o ambiente Blockly, que pode ser acessado pelo endereço $<$ https://blockly.games/ $>$ que pode ser utilizado como uma plataforma educacional, ou como parte de um sistema de jogos. A sua escolha ocorreu pela simplicidade e potencial de extensão da ferramenta. Apresentou-se então um framework (templates usados por um desenvolvedor) para desenvolvimento de jogos digitais voltados para o ensino de lógica de programação.

Falcão e Barbosa (2015) discutem, em sua pesquisa, o jogo Lightbot que tem o intuito de apresentar ao jogador desafios de lógica de programação com um objetivo simples: movimentar um robô de um ponto até o ladrilho azul e acender a lâmpada ali presente, deixando assim o ladrilho amarelo. Começam com poucos comandos, para que o jogador entenda cada funcionalidade e à medida que o jogador vai passando de fases, os desafios vão ficando mais difíceis, entrando em questão as propostas de aprendizado de controle de fluxo, de funções, de laços e maiores desafios de lógica com uma quantidade limitada de comandos que o jogador possui para resolver o problema. O desenvolvimento do "código" é sempre feito arrastando e soltando os blocos de comandos para que o robô se movimente. $\mathrm{O}$ artigo concluiu que o ambiente de experimentação de comandos com visualização dos resultados não foi suficiente para guiar as crianças, fazendo-se necessária a mediação de um facilitador.

Martins et al. (2016), em seu artigo, mostraram o resultado do trabalho com crianças do Ensino Fundamental, em que foram abordadas noções básicas de lógica, algoritmo e programação, por meio de aulas teóricas e práticas, utilizando 
jogos do site "Racha Cuca" e a programação com o Scratch, para posteriormente as crianças utilizarem os conhecimentos adquiridos na programação dos robôs Lego Mindstorm - um software livre e fácil de ser manipulado, que apresenta missões e uma interface de programação simples. Além de obter uma forma lúdica de promover a iniciação à robótica, os alunos puderam, assim, programar, inserir a implementação feita efetuando o download para a memória do robô e realizar os testes. A partir do uso dos recursos foram montados os robôs e, posteriormente, programados na própria plataforma. Nesta fase do projeto houve maior empolgação dos envolvidos:

Foi visível a reação de felicidade das crianças a cada vez que o robô realizava uma tarefa proposta. No decorrer do processo, observou-se maior dificuldade dos alunos para entender os conceitos. Já na parte prática, os discentes adoraram e apresentaram um ótimo rendimento (MARTINS et al., 2016, p.39).

No artigo de Ribeiro e Melo (2017) ficou evidenciado que o ambiente Scratch foi utilizado para o desenvolvimento do pensamento computacional e lógico, sem haver necessariamente a preocupação com o desenvolvimento sistemático de um produto de software com crianças. Percebeuse a evolução das crianças com relação aos conhecimentos em programação, lógica e resolução de problemas.

As crianças envolvidas demonstraram interesse em continuar participando no desenvolvimento de tecnologia e de estudos semelhantes, evidenciando um sentimento significativo de coautoria em tudo aquilo o que foi produzido (RIBEIRO; MELO, 2017, p.1033).

O uso do software Scratch foi desenvolvido por um grupo de pesquisadores do Lifelong Kindergarten no Media Lab do Massachusetts Institute of Technology - MIT coordenado pelo professor doutor Mitchel Resnick. A programação com este software é bastante acessível para a criança, que pode construir programas (figuras, cenários, narrativas, histórias interativas, jogos, sons e animações) utilizando o encadeamento de blocos lógicos de comandos e de operações. Esse processo de construção pode ser compartilhado de maneira online com outros alunos, permitindo com isso a construção em grupo de um produto (o programa). Este software tem sido utilizado em vários contextos de escolas em diferentes países, pois é um dos recursos que favorecem ao aluno, desde o início da escolarização, aprender de uma nova forma e a desenvolver o raciocínio lógico.

O artigo dos autores Guarda e Goulart (2018) buscou estudar as alternativas para a construção de um processo de ensino e aprendizagem "gamificado" visando contribuir para a inserção de novos métodos e melhoria do rendimento escolar sob a ótica do pensamento computacional. Foram construídos objetos de aprendizagem com materiais acessíveis para trabalhar a inserção de habilidades como montagem de sequências lógicas, lógica de programação, criptografia, entre outros. A iniciativa se mostrou interessante, uma vez que os estudantes se identificaram e se envolveram emocionalmente, propiciando uma aprendizagem significativa, principalmente, na disciplina de Matemática que, na maioria das vezes, apresentam maior dificuldade no aprendizado.

Assim, percebe-se que o uso das tecnologias computacionais de caráter lúdico na educação com as crianças pode trazer benefícios para aprendizagem inquestionável e que estas ferramentas ajudam não só no raciocínio lógico das crianças como também incentiva a criatividade e a resolução de problemas.

\section{Nível 2: Lógica de programação no Ensino médio}

A temática da computação pode ser vista na BNCC (Brasil, 2018, p. 474), que trata do seu uso no Ensino Médio das escolas brasileiras. No trecho "As tecnologias digitais e a computação" é apresentado como a computação quanto às tecnologias digitais de informação e comunicação (TDIC) estão cada vez mais presentes na vida de todos, não somente nos escritórios ou nas escolas, mas também nos bolsos, nas cozinhas, nos automóveis, nas roupas etc. A computação e as tecnologias digitais são tematizadas, tanto no que diz respeito aos conhecimentos e habilidades quanto às atitudes e valores, entre esses:

Pensamento computacional: envolve as capacidades de compreender, analisar, definir, modelar, resolver, comparar e automatizar problemas e suas soluções, de forma metódica e sistemática, por meio do desenvolvimento de algoritmos. Portanto, na BNCC dessa etapa, o foco passa a estar no reconhecimento das potencialidades das tecnologias digitais para a realização de uma série de atividades relacionadas a todas as áreas do conhecimento, a diversas práticas sociais e ao mundo do trabalho. São definidas competências e habilidades, nas diferentes áreas, que permitem aos estudantes (BRASIL, 2018, p. 475).

Relacionado ao Ensino Médio foram encontrados cinco artigos, que tratam deste assunto conforme Quadro 5:

Quadro 5 - Artigos relacionados ao Ensino Médio

\begin{tabular}{|l|l|}
\hline \multicolumn{1}{|c|}{ Autores } & \multicolumn{1}{c|}{ Tema } \\
\hline $\begin{array}{l}\text { Fernanda Mota, Nathan } \\
\text { Ribeiro, Leonardo } \\
\text { Emmendorfer, Paulo Butzen, } \\
\text { Karina Machado, Diana } \\
\text { Adamatti }\end{array}$ & $\begin{array}{l}\text { Desenvolvendo o Raciocínio } \\
\text { Lógico no Ensino Médio: } \\
\text { uma proposta utilizando a } \\
\text { ferramenta Scratch }\end{array}$ \\
\hline $\begin{array}{l}\text { Narúsci Bastos, Diana } \\
\text { Adamatti, Fernanda } \\
\text { Antoniolo }\end{array}$ & $\begin{array}{l}\text { Ensino de Lógica de } \\
\text { Programação no Ensino } \\
\text { Médio e suas implicações na }\end{array}$ \\
\hline $\begin{array}{l}\text { Glívia Barbosa, Ismael } \\
\text { Santana, Flávio Coutinho, } \\
\text { Pedro Froes, Rafael Barbosa, } \\
\text { Felipe Freitas, Vitória M. } \\
\text { P. do Nascimento, Mariana } \\
\text { Bulgarelli, Gustavo Marques, } \\
\text { João Renan, Paula Ribeiro, } \\
\text { Joice Sena, Daniel Franco }\end{array}$ & $\begin{array}{l}\text { Play(code): uma proposta } \\
\text { para estimular o aprendizado } \\
\text { de Lógica de Programação }\end{array}$ \\
\hline Fonte: dados da pesquisa.
\end{tabular}

No artigo de Mota et al (2014), os autores discutem como a computação pode ir além do uso do computador para a 
realização de atividades rotineiras como acesso à internet e elaboração de textos e trabalhos.

O computador pode ser utilizado no desenvolvimento de novos programas ou jogos com diferentes funcionalidades, uma atividade que envolve conhecimentos de lógica de programação que podem ser aplicados em várias áreas, não se limitando a informática. A integração da lógica de programação no ensino básico e profissional pode auxiliar na resolução de problemas de forma estruturada e racional (MOTA et al., 2014, p.377).

Foi desenvolvido um curso de lógica de programação, por meio do software Scratch, para adolescentes do Ensino Médio em uma escola pública. O projeto ainda inacabado apresentou, em seus primeiros resultados, o interesse dos alunos participantes em darem continuidade ao estudo de lógica de programação. Esses alunos têm criado com o Scratch jogos para a solução de problemas de Matemática. Tais soluções de problemas se diferem daquelas que anteriormente eram resolvidos de forma tradicional, sem envolver o aluno no processo de aprender.

Na pesquisa de Bastos et al (2015), os autores buscaram estudar como a lógica computacional poderia interferir no raciocínio lógico dos alunos do Ensino Médio. Foi necessário identificar possíveis mudanças na fisiologia cerebral antes e após os treinamentos na área de lógica e caracterizar a atividade cerebral durante a realização do teste de lógica antes e depois do treinamento.

Observou-se que houve alterações na fisiologia cerebral, confirmando assim as hipóteses propostas pela pesquisa: (1) os alunos sem treinamento em raciocínio lógico tendem a ativar diversas áreas cerebrais; (2) alunos com treinamento em raciocínio lógico ativam áreas cerebrais específicas.

Barbosa et al (2018) apresentaram uma pesquisa que buscou estimular o aprendizado de lógica de programação, por meio de uma plataforma online gamificada e colaborativa, denominada Play(code). O objetivo era de oferecer um ambiente de ensino que pudesse estimular o aprendizado, autodidata ou mediado por um tutor, por meio de um computador pessoal ou dispositivo móvel. A plataforma se encontra em desenvolvimento, mas se mostrou bastante promissora em relação aos primeiros testes.

Dessa forma, pode-se concluir que a utilização da Lógica da Programação neste período escolar interfere, positivamente, nos aspectos cognitivos relacionados, principalmente, a solução de problemas através da utilização de jogos, como o Scratch, e que podem contribuir para que os alunos desempenhem melhor os aspectos relacionados ao raciocínio lógico.

Por fim, apresenta-se como a Lógica de Programação pode ajudar os estudantes do Ensino Superior a melhorar suas capacidades cognitivas e o desempenho nas disciplinas relacionadas com a Matemática e Programação.

\section{Nível 3: Lógica de programação no Ensino Superior}

A disciplina de Algoritmos é a base essencial na formação dos alunos dos cursos da área de Computação. É o primeiro contato direto dos alunos com ambientes, que trabalham os conceitos de raciocínio lógico, matemática e programação tanto de instituições públicas quanto privadas (JESUS; BRITO, 2009).

Segundo a Sociedade Brasileira de Computação (2003), a estrutura curricular básica dos cursos de Computação apresenta em sua composição uma área de formação básica denominada "Ciência da Computação", que é subdividida em três partes: programação, computação e algoritmos e arquitetura de computadores. Tal estruturação demonstra de forma clara a real importância que é direcionada aos algoritmos no currículo de formação discente na área em foco (PAULA et al., 2019, p.152).

Este nível trouxe o maior número de artigos relacionados com a temática, conforme o Quadro 6.

Quadro 6 - Artigos relacionados ao Ensino Superior

\begin{tabular}{|l|}
\hline \multicolumn{1}{|c|}{ Autores } \\
\hline Cárlisson Galdino, \\
Sebastião Neto, Evandro \\
Costa
\end{tabular}

Leandro Nagano e Alexandre Direne.

Erico Amaral, Alex Camargo, Marina Gomes, Cesar Huegel Richa, Liliane Becker KidCoder: uma Proposta de Ensino de Programação de forma Lúdica

\begin{tabular}{l}
\hline $\begin{array}{l}\text { Brenda Barbosa, Sandro } \\
\text { Silva, Bruno Sousa }\end{array}$ \\
\\
Carla Rodriguez, Rafaela \\
Vilela da Rocha, Denise \\
Goya, Mirtha Fernández \\
Venero, Francisco \\
Zampirolli
\end{tabular}

Carla Rodriguez, Denise

Goya, Mirtha Lina

Fernandéz Venero, Cláudia Abrão

Lord of Code: uma Ferramenta de Apoio ao Ensino da Programação

Ensino de Lógica de Programação baseado na indução-dedução através de exemplos

ALGO+ Uma ferramenta para o apoio ao ensino de Algoritmos e Programação para alunos iniciantes

Tri-Logic Proposta Lúdica

Gamificada para o Ensino e Aprendizagem da Lógica de Programação com o Uso da Mineração de Dados como Ferramenta de Auxílio ao Professor

Critérios para inserção

de estratégias cognitivas e metacognitivas no

desenvolvimento de lógica de programação em ambientes virtuais de aprendizagem

Met@Aprendiz@gem: estratégias metacognitivas e recursos pedagógicos para apoiar a construção do pensamento lógico

Ensino de Algoritmos e Lógica de

Luiza Engler Stadelhofer, Isabela Gasparini

Programação para os Diferentes

Cursos: um Mapeamento Sistemático da Literatura

Captura e Visualizacão das

Êrica Peters do Carmo, Isabela Gasparini, Elaine Oliveira

Trajetórias de Aprendizagem como Ferramentas para a Análise do Comportamento dos Estudantes em um Ambiente Adaptativo Educacional

Predição do rendimento dos

Renata Barros, Orivaldo Santana Jr, Igor Silva, Luana dos Santos, Vilson Rodrigues Câmara Neto alunos em lógica de programação com base no desempenho das disciplinas do primeiro período do Curso de Ciências e Tecnologia utilizando técnicas de mineração de dados

Fonte: dados da pesquisa. 
Galdino et al (2015) apresentam uma proposta de um jogo na forma de um ambiente de prática, para motivar alunos, apresentando desafios com dificuldade gradual, envoltos em um enredo imersivo. Esta metodologia visa diminuir a evasão dos cursos de Ciência da Computação, identificadas pela dificuldade dos alunos em compreenderem conteúdos ligados à lógica de programação. Os autores têm o propósito de proporcionar aos alunos um jogo denominado Kidcoder, no qual o ambiente para estímulo à programação ocorre na solução de conflitos e desafios: os quais serão resolvidos através de programação. O trabalho ainda não está concluído, visto que se pretende, com o projeto, oferecer aos alunos de Programação mais uma ferramenta que os motive e lhes permita praticar codificação e montagem de algoritmos.

Café et al (2017) propuseram o desenvolvimento de um jogo, chamado Lord of Code (LOC) para auxiliar na linguagem Java de programação e na solução de problemas em algoritmos computacionais. O objetivo é que o jogador faça suas análises sobre um código Java e indique qual resultado será retornado pelo algoritmo ou identifique qual trecho apresenta erro. As regras do jogo consistem em o jogador digitar a resposta correta, se ele acertar as questões então marcará pontos e acumulará ajudas que poderão ser usadas para as questões seguintes. O trabalho apenas propõe uma ferramenta que auxilie no ensino de lógica de programação através de um protótipo a ser realizado futuramente.

Nagano e Direne (2016) corroboram com as pesquisas anteriores de que há muita dificuldade dos alunos na compreensão da disciplina de lógica de programação, sendo este um dos motivos para a evasão nos Cursos de Computação. Para auxiliar no aprendizado dessa disciplina, um sistema de tutor inteligente foi projetado abordando conceitos pedagógicos e computacionais. A pesquisa bibliográfica procurou mostrar como os pressupostos de Vigotsky, especialmente a mediação, baseada no conceito da zona de desenvolvimento proximal e nas ideias de Felder-Silverman envolvendo as cinco dimensões de aprendizagem (ativosreflexivos; sensoriais-intuitivos; visuais-verbais; indutivosdedutivos; sequenciais-globais) podem auxiliar no processo de aprendizagem da lógica de programação.

Já Amaral et al (2017) sugerem a adoção de um ambiente virtual de aprendizagem (AVA), no qual professores e alunos pudessem interagir e permitir a autonomia no desenvolvimento dos estudos. O ambiente disponibiliza um conjunto de recursos midiáticos, que busca atender os diferentes estilos de aprendizagem, baseados no Ciclo de Aprendizagem Experiencial de Kolb. O desenvolvimento de uma solução para apoio ao processo de ensino e aprendizagem da disciplina de Algoritmos e Programação, a qual foi denominada Algo+. A fim de permitir a autonomia do aluno no desenvolvimento de seus estudos, o Portal disponibiliza um conjunto de recursos midiáticos, que objetiva atender os diferentes estilos de aprendizagem.
A utilização do Algo+ por um conjunto de estudantes possibilitou concluir que, efetivamente, sua organização permitiu que os discentes realizassem seus estudos de forma satisfatória. Um ponto interessante a ser descrito, refere-se ao fato da característica híbrida que a estrutura deste software concede, visto que propicia ao professor um recurso de acompanhamento de seus alunos, porém também pode ser utilizado como ferramenta autônoma para discente que desejem aprender programação (AMARAL et al, 2017, p.1685).

Barbosa, B. et al (2017) sugerem o desenvolvimento de ambientes gamificados como ferramenta de ensino e aprendizagem de lógica de programação. Iniciou-se o desenvolvimento do Tri-Logic, que propõe um ambiente de apoio ao ensino desta disciplina, aliando aos jogos eletrônicos o monitoramento online de suas etapas, por meio do uso de técnicas de mineração de dados educacionais. Ao final dos experimentos, os alunos se sentiram motivados a seguirem aprendendo lógica de programação e um novo protótipo TriLogic poderá ser desenvolvido visando melhorias em seu desempenho.

Rodriguez et al (2018) apresentam o resultado de uma pesquisa em que mostram que os alunos da disciplina de Processamento de Informação possuem grande dificuldade de aprendizagem. A taxa de reprovação e evasão se deve muito a esta disciplina que contempla, principalmente, lógica de programação. Foi sugerida uma proposta, sem aplicação até o momento, para aliar o uso de estratégias metacognitivas e recursos pedagógicos para favorecer o engajamento e a motivação dos estudantes e orientar o seu percurso de aprendizagem. O programa deve priorizar o uso de objetos de aprendizagem como resumos, infográficos, animações e jogos digitais aos quais se pretende incluir roteiros que auxiliem os alunos no planejamento, avaliação e regulação da aprendizagem.

Outro estudo de Rodriguez et al (2017) também parte do princípio do uso de estratégias capazes de aumentar a motivação e o engajamento ampliando o desempenho dos alunos no aprendizado dos conteúdos no ensino a distância. O projeto Met@Aprendiz@gem mira apresentar recursos e estratégias de ensino e aprendizagem para apoiar a construção do pensamento lógico e propor iniciativas pedagógicas capazes de mobilizar as estratégias cognitivas e metacognitivas.

[...] artigo descreveu critérios que apoiam o uso das estratégias cognitivas e metacognitivas pelo aluno no desenvolvimento de lógica de programação, e apresenta checklists que relacionam essas estratégias de aprendizagem com recursos e ferramentas que podem auxiliar o professor a promover o uso dessas estratégias em ambientes computacionais (RODRIGUEZ et al., 2017, p.1190).

Stadelhofer e Gasparini (2019) fazem um mapeamento sistemático de literatura apresentando um parâmetro da Educação Superior de disciplinas introdutórias de programação no contexto de cursos não pertencentes à área de computação. Entre os resultados se pode constatar que as metodologias de ensino comuns, como aulas práticas e de laboratório, são 
recorrentes nestes cursos e que há necessidade de uma revisão nas formas de construir o conhecimento.

Carmo et al (2019) objetivam, em sua pesquisa, utilizar as trajetórias dos estudantes de um minicurso de algoritmos e lógica de programação para analisar o seu padrão navegacional. $\mathrm{O}$ ambiente virtual de aprendizagem utilizado neste trabalho para a extração dos dados referentes às trajetórias dos estudantes foi o AdaptWeb, um ambiente adaptativo de ensino na Web capaz de adaptar seu o conteúdo, apresentação e navegação de acordo com as preferências e características do usuário. Assim, a partir dos dados dos estudantes, suas trajetórias foram capturadas e representadas visualmente. Os resultados obtidos mostram que os estudantes preferem realizar uma navegação linear baseada em conceitos. Contudo, ainda não foi possível traçar uma relação entre os recursos educacionais acessados pelos estudantes e seu desempenho.

Barros et al. (2019) propõem a utilização de técnicas de Mineração de Dados Educacionais para tentar predizer o desempenho dos alunos na disciplina de Lógica de Programação nos anos iniciais da área de tecnologia. Foram utilizados algoritmos de aprendizagem de máquina treinados para verificar a situação final do aluno nas disciplinas do primeiro semestre para prever a sua situação ou desempenho em uma disciplina do segundo semestre.

Através da linguagem Python e suas bibliotecas Pandas e scikit-learn, obteve-se precisão de até $72 \%$, utilizando um conjunto de seis atributos de entrada: As médias das notas das disciplinas de Pré-Cálculo, Cálculo I, Química Geral, Práticas de Leitura e Escrita I, Ciência, Tecnologia e Sociedade e Vetores e Geometria Analítica obtidas no primeiro período do curso (BARROS et al., 2019, p.1498).

Pode-se concluir que as pesquisas realizadas sobre Lógica de Programação no Ensino Superior procuram, em grande parte, tentar solucionar a dificuldade que os alunos possuem em aprender conteúdos relacionados à Lógica. O foco dos estudos está em facilitar a aprendizagem destes alunos oferecendo ferramentas, como a gamificação, para incentiválos a não desistir dos cursos, principalmente, aqueles ligados à computação.

\section{Conclusão}

Neste artigo se apresenta uma revisão sistemática e ordenada da literatura sobre a Lógica da Programação em artigos científicos publicados nos últimos seis anos no repositório do Simpósio Brasileiro de Informática na Educação (SBIE). Na busca inicial, vinte e duas publicações foram encontradas e dezoito foram utilizadas após a aplicação dos critérios de exclusão e inclusão. Os artigos tratam do processo de ensino da Lógica da Programação nos três níveis de Educação do sistema brasileiro, ou seja, do Ensino Fundamental, Ensino Médio e o Ensino Superior. Podese constatar que o intuito de incluir atividades voltadas à programação nos anos iniciais de escolaridade, ou seja, do
Ensino Fundamental, é para que as crianças possam vivenciar uma nova forma de aprender "ensinando a máquina" de modo a favorecer o desenvolvimento do pensamento lógico, estimular sua criatividade e desenvolver a capacidade de resolução de problemas. No Ensino Médio, apesar de ter encontrado apenas um artigo, o mesmo trata de uma pesquisa que também propõe o uso da Lógica Computacional para o desenvolvimento do raciocínio lógico dos estudantes. Já no Ensino Superior, em que a maioria das pesquisas foi retratada se pode perceber a preocupação dos pesquisadores em identificar e propor novas metodologias de ensino, principalmente, para os cursos ligados à computação, para que não ocorra o grande número de evasão que é identificada nos dias de hoje. São propostas de tutores inteligentes, ambientes virtuais de aprendizagem e gamificação.

\section{Referências}

AMARAL, É. et al. ALGO+ Uma ferramenta para o apoio ao ensino de Algoritmos e Programação para alunos iniciantes. In: CONGRESSO BRASILEIRO DE INFORMÁTICA NA EDUCAÇÃO, 2017, Recife. Anais do SBIE [...]. Porto Alegre: SBC, 2017.

BARBOSA, B. et al. Tri-Logic - Proposta Lúdica Gamificada para o Ensino e Aprendizagem da Lógica de Programação com o Uso da Mineração de Dados como Ferramenta de Auxílio ao Professor. In: CONGRESSO BRASILEIRO DE INFORMÁTICA NA EDUCAÇÃO, 2017, Recife. Anais do SBIE [...]. Porto Alegre: SBC, 2017..

BARBOSA, G.A.R. et al. Play(code): Uma proposta para estimular o aprendizado de Lógica de Programação. In: CONGRESSO BRASILEIRO DE INFORMÁTICA NA EDUCAÇÃO, 2018, Fortaleza. Anais do SBIE [...]. Porto Alegre: SBC, 2018.

BARROS, R.P. et al. Predição do rendimento dos alunos em lógica de programação com base no desempenho das disciplinas do primeiro período do curso de ciências e tecnologia utilizando técnicas de mineração de dados. In: SIMPÓSIO BRASILEIRO DE INFORMÁTICA NA EDUCAÇÃO (SBIE 2019), Brasília, p. 1491-1500, 11 nov. 2019.

BASTOS, N.S.; ADAMATTI, D.F.; CARVALHO, F.A. Ensino de Lógica de Programação no Ensino Médio e suas implicações na Neurociências. In: CONGRESSO BRASILEIRO DE INFORMÁTICA NA EDUCAÇÃO; CONFERÊNCIA LATINO-AMERICANA DE OBJETOS E TECNOLOGIAS DE APRENDIZAGEM, 2015, Maceió. Anais... Porto Alegre: SBC, 2015.

BRASIL. Base Nacional Comum Curricular (BNCC). As Tecnologias Digitais e a Computação. Brasília: MEC, 2018.

CARMO, Ê.P.; GASPARINI, I.; OLIVEIRA, E.H.T. Captura e visualização das trajetórias de aprendizagem como ferramentas para a análise do comportamento dos estudantes em um ambiente adaptativo educacional. In: SIMPÓSIO BRASILEIRO DE INFORMÁTICA NA EDUCAÇÃO. Brasília, p. 3019-318, 11 nov. 2019.

FALCÃO, T.P.; BARBOSA, R.S. “Aperta o Play!” Análise da Interação Exploratória em um Jogo Baseado em Pensamento Computacional. In: CONGRESSO BRASILEIRO DE INFORMÁTICA NA EDUCAÇÃO; CONFERÊNCIA LATINO-AMERICANA DE OBJETOS E TECNOLOGIAS DE APRENDIZAGEM, 2015, Maceió. Anais.... Porto Alegre: SBC, 2015. 
FORBELLONE, A.L.V.; EBERSPACHER, H.F. Lógica de Programação: a construção de algoritmos e estruturas de dados. São Paulo: Prentice Hall, 2005.

GALDINO, C.B.T.; SILVA NETO, S.R.; COSTA, E.B. KidCoder: uma proposta de ensino de programação de forma lúdica. In: CONGRESSO BRASILEIRO DE INFORMÁTICA NA EDUCAÇÃO; CONFERÊNCIA LATINO-AMERICANA DE OBJETOS E TECNOLOGIAS DE APRENDIZAGEM, 2015, Maceió. Anais... Porto Alegre: SBC, 2015.

GUARDA, G.F.; GOULART, I.F. Jogos Lúdicos sob a ótica do Pensamento Computacional: Experiências do Projeto Logicamente. In: CONGRESSO BRASILEIRO DE INFORMÁTICA NA EDUCAÇÃO, 2018, Fortaleza. Anais... Porto Alegre: SBC, 2018.

JESUS, A. DE; BRITO, G. S. Concepção de ensino-aprendizagem de algoritmos e programação de computadores: a prática docente. Rev. Varia Scie., v.9, n.16, p. 149-158, 2009.

MARTINS, L.A.S. et al. Ensinando lógica de programação aplicada à robótica para alunos do Ensino Fundamental. In: CONGRESSO BRASILEIRO DE INFORMÁTICA NA EDUCAÇÃO, 2016, Uberlândia. Anais... Porto Alegre: SBC, 2016.

MEDEIROS, T.; BRASIL, P.; ARANHA, E. Um framework para criação de jogos voltados para o ensino de lógica de programação.In: SIMPÓSIO BRASILEIRO DE INFORMÁTICA NA EDUCAÇÃO. 2014, Dourados. Anais... Porto Alegre: SBC, 2014.

MOTA, F.P. et al. Desenvolvendo o Raciocínio Lógico no Ensino Médio: uma proposta utilizando a ferramenta Scratch. In: SIMPÓSIO BRASILEIRO DE INFORMÁTICA NA EDUCAÇÃO, 2014, Dourados. Anais... Porto Alegre: SBC, 2014.

NAGANO, L.H.; DIRENE, A.I. Ensino de lógica de programação baseado na indução-dedução através de exemplos. In: CONGRESSO BRASILEIRO DE INFORMÁTICA NA EDUCAÇÃO, 2016, Uberlândia. Anais... Porto Alegre: SBC, 2016

PAPERT, S. Logo: computadores e educação. São Paulo: Brasiliense, 1985.

PAPERT, S. A máquina das crianças: repensando a escola na era da informática. Porto Alegre: Artes Médicas, 1994.

PAULA, E.F. et al. Educação: interlocutores, diálogos e reflexões na contemporaneidade. São Paulo: Pimenta Cultural, 2019.

PRADO, M.E.B.B. et al. Pensamento computacional e atividade de programação: perspectivas para o ensino de matemática. Rev. Sergipana Matem. Educ. Matem., v.5, n.2, p 195-208, 2020.
PUGA, S.; RISSETTI, G. Lógica de programação e estrutura de dados, com aplicações em Java. São Paulo: Pearson Prentice Hall, 2009.

RAMOS, A.; FARIA, P.; FARIA, Á. Revisão sistemática de literatura: contributo para a inovação na investigação em Ciências da Educação. Rev. Diál. Educ., v.14, n.41, 2014.

RIBEIRO, S.S.; MELO,A.M. Um método para o desenvolvimento de software com crianças utilizando o Ambiente Scratch. In CONGRESSO BRASILEIRO DE INFORMÁTICA NA EDUCAÇÃO, 2017, Recife. Anais... Porto Alegre: SBC, 2017.

RODRIGUEZ, C.L. et al. Critérios para inserção de estratégias cognitivas e metacognitivas no desenvolvimento de lógica de programação em ambientes virtuais de aprendizagem. In: CONGRESSO BRASILEIRO DE INFORMÁTICA NA EDUCAÇÃO, 2018, Fortaleza. Anais... Porto Alegre: SBC, 2018.

RODRIGUEZ, C. et al. Met@ Aprendiz@gem: estratégias metacognitivas e recursos diferenciados para apoiar a construção do pensamento lógico. In: CONGRESSO BRASILEIRO DE INFORMÁTICA NA EDUCAÇÃO, 2017, Recife. Anais... Porto Alegre: SBC, 2017.

SAMPIERI, R.H.; COLLADO, C.F.; LUCIO, M.P.B. Metodologia de pesquisa. Porto Alegre: Penso, 2013.

SOCIEDADE BRASILEIRA DE COMPUTAÇÃO. Currículo de referência da SBC para cursos de graduação

em computação e informática. 2003. Disponível em: www.sbc. org.br/educacao. Acesso em: 10 maio 2020.

SOUZA, M.S.C. et al. Lord of Code: uma ferramenta de apoio ao ensino de programação. In: CONGRESSO BRASILEIRO DE INFORMÁTICA NA EDUCAÇÃO, 2016, Uberlândia. Anais... Porto Alegre: SBC, 2016.

STADELHOFER, L.E.; GASPARINI, I. Ensino de algoritmos e lógica de programação para os diferentes cursos: um mapeamento sistemático da literatura. In: CONGRESSO BRASILEIRO DE INFORMÁTICA NA EDUCAÇÃO, 2018, Fortaleza. Anais... Porto Alegre: SBC, 2018.

VALENTE, J.A. Integração do pensamento computacional no currículo da educação básica: diferentes estratégias usadas e questões de formação de professores e avaliação do aluno. Rev. eCurriculum, v.14, n.3, p.864-897, 2016.

WING, J.M. Computational thinking. Communications ACM, v.49, n.3, p.33-35, 2006. 